\title{
Astrometric planet searches with SIM PlanetQuest
}

\author{
C. A. Beichman ${ }^{1}$, S. C. Unwin ${ }^{2}$, M. Shao ${ }^{2}$, A. M. Tanner ${ }^{1,2}$, \\ J. H. Catanzarite ${ }^{2}$ and G. W. Marcy ${ }^{3}$ \\ ${ }^{1}$ Michelson Science Center, California Institute of Technology, 770 S. Wilson Ave., Pasadena, \\ CA 91125 \\ email: chas@ipac.caltech.edu \\ ${ }^{2}$ Jet Propulsion Laboratory, California Institute of Technology, 4800 Oak Grove Drive, \\ Pasadena, CA 91109 \\ ${ }^{3}$ University of California, Berkeley, 417 Campbell Hall, Berkeley, CA 94720
}

\begin{abstract}
SIM will search for planets with masses as small as the Earth's orbiting in the 'habitable zones' around more than 100 of the nearest stars and could discover many dozen if Earth-like planets are common. With a planned "Deep Survey" of 100-450 stars (depending on desired mass sensitivity) SIM will search for terrestrial planets around all of the candidate target stars for future direct detection missions such as Terrestrial Planet Finder and Darwin. SIM's "Broad Survey" of 2100 stars will characterize single and multiple-planet systems around a wide variety of stellar types, including many now inaccessible with the radial velocity technique. In particular, SIM will search for planets around young stars providing insights into how planetary systems are born and evolve with time.
\end{abstract}

Keywords. astrometry, planets and satellites: formation, solar system: formation

\section{Introduction}

The existence of planets orbiting around other stars has been transformed from a philosophical question (Epicurus, ca. 300 BCE) and a scientific aspiration (Struve 1952) to an astronomical industry with more than 250 planets discovered via radial velocity measurements (Mayor et al. 1995; Marcy et al. 2005; Butler et al. 2006), transits (Henry et al. 2000) and microlensing (Beaulieu et al 2006). Each technique contributes to the long term goal of detecting and characterizing earth-like planets and ultimately finding signatures of life beyond our solar system (Beichman et al. 2007). This paper highlights astrometric searches for planets and the possibilities for developing a fuller understanding of the existence, formation and evolution of planetary systems using the Space Interferometer Mission (SIM PlanetQuest). An overview and a complete description of SIM's astrometric goals in all areas of astrophysics can be found in Shao et al. (this volume) and in Unwin et al. (2007), respectively. As will be described below, SIM will carry out three distinct programs: a deep search for rocky planets, a broad search for more massive planets orbiting a wide variety of host stars, and a search for planets around young stars. A modestly descoped version of SIM, nominally called SIM-Lite, can achieve the same measurement goals as SIM/PlanetQuest but for only half the number of objects.

\section{The search for potentially habitable planets}

SIM measures three crucial characteristics of a planet that suggest the circumstances of its birth, define its long term fate, and hint at its suitability for the existence of life: mass, 

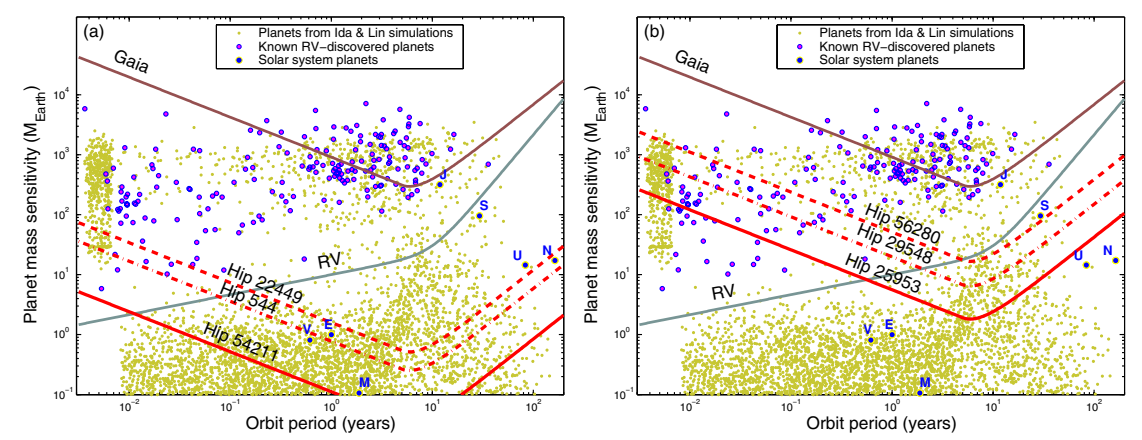

Figure 1. a, left $)$ The discovery space for rocky Earth-like $\left(\sim 1-10 M_{\oplus}\right)$ planets in the habitable zone ( 0.7-1.5 AU for a G star), for an "Earth Analog Survey" of 129 stars; b, right) a comparable plot for the "Broad Survey" obtained with $4 \mu$ as accuracy. In both cases, the small dots represent a theoretical planet distribution Ida \& Lin (2005) for planets of $0.1-3000 M_{\oplus}$. Exoplanets discovered before early 2007 and with semimajor axes $>0.03 \mathrm{AU}$ are shown as filled circles. Labeled curves represent the estimated sensitivity limits of indirect detection methods: for radial velocity method (RV at $1 \mathrm{~m} \mathrm{~s}^{-1}$ ), and astrometry with SIM and Gaia. The SIM sensitivity in this space is a broad band, defined by the three lowest curves (labeled with specific Hipparcos star numbers). The lowest curve shows the 'best' star (as computed from star mass and distance); the middle curve represents the median star; and the upper curve shows the least favorable star in the sample. Also shown is the effective sensitivity of Gaia for stars at $50 \mathrm{pc}$, a typical distance for Gaia targets.

the size and shape of its orbit, and its orbital inclination (where the latter quantity allows us to assess the critical question of co-planarity relative to other planets in the system). Perhaps the most important characteristic is mass. During the formation phase, an object with a mass greater $10 \mathrm{M}_{\oplus}$ will probably rapidly accrete enough volatiles to become an icy or gas giant. An object with a mass less than that of Mars is unlikely to retain volatiles to form an atmosphere or harbor oceans of liquid water. A planet's semi-major axis is also critical: too close to its parent star, stellar insolation and a runaway greenhouse will produce a Cytherean Hell, too far from the star will result in a world of perpetual ice. Secondary effects such as high eccentricity or interactions with other planets will adversely affect short and long term climatic stability and even continued membership in the planetary system. By finding planets of terrestrial mass in the habitable zones of their parent stars (Kasting et al. 1993) SIM will make a dramatic step toward realizing the goal of identifying other habitable worlds (Beichman et al. 2007).

The radial velocity $(\mathrm{RV})$ technique will have great difficulty in detecting planets of $1 M_{\oplus}$ orbiting solar type stars near $1 \mathrm{AU}$, as the RV signature of an earth analog is $\sim 0.1 \mathrm{~m} \mathrm{~s}^{-1}$ compared with stellar surface jitter of $\sim 1 \mathrm{~m} \mathrm{~s}^{-1}$. While ESA's Gaia mission will detect many exoplanets (Lindegren and Sozzetti, this volume), Gaia's discovery space is that of gas-giant planets (Fig. 1). Furthermore, Gaia will achieve its highest precision for stars only fainter than $\sim 7$ mag due to detector effects, thereby eliminating the brightest, closest stars from the search for the lowest mass planets. SIM offers a unique opportunity to detect Earth analogs, planets of one Earth mass in the habitable zones of nearby Solar-type stars.

\subsection{Astrometric detection of terrestrial planets}

SIM's narrow-angle astrometry of each target star will be made relative to at least 3 reference stars selected to surround the target star within $1^{\circ} \sim 5^{\circ}$. The reference stars are K giants brighter than $V=10 \mathrm{mag}$, within roughly $600 \mathrm{pc}$, so that the astrometric 'noise' due from planets orbiting their reference stars is minimized (Frink et al. 2001). A single 
Table 1. Planet Mass-Limited Surveys with SIM

\begin{tabular}{lcc}
\hline & Mass sensitivity & Number of stars surveyed \\
\hline Survey 1 & $1.0 M_{\oplus}$ & 129 \\
Survey 2 & $2.0 M_{\oplus}$ & 297 \\
Survey 3 & $3.0 M_{\oplus}$ & 465 \\
\hline
\end{tabular}

measurement will achieve $0.85 \mu$ as differential measurement precision for $V=7$ stars, including instrumental and photon-limited errors compared with the $0.3 \mu$ as signature of an Earth analog orbiting a solar type stars at 10 pc. Recent laboratory measurements indicate that SIM's systematic noise floor is below $0.1 \mu$ as after many repeated measurements, opening up the possibility of detecting Earth mass planets around over 100 of the closest stars. Starspots and other manifestations of stellar variability can affect the astrometric centroid resulting in a jitter similar to the spectroscopic noise that limits radial velocity precision. Models of astrometric jitter based on many years of precision photometry of the Sun indicate that these effects will not be the primary limitation for the detection of terrestrial planets around quiescent solar type stars (Unwin et al. 2007; Catanzarite, Shao \& Law, in preparation). More realistic modeling for a variety of spectral types and stellar ages will be possible using data from the CoRoT and Kepler satellites.

\subsection{The SIM Deep Survey}

In an "Earth Analog Survey" SIM will study up to several hundred stars located within $30 \mathrm{pc}$ in a manner designed to detect the lowest possible mass planets in the habitable zone of each star, $r_{H Z} \sim 1\left(L / L_{\odot}\right)^{0.5}$ AU. We allocate to each target enough integration time to allow a planet of $1 M_{\oplus}$ to be detected at the radius of its mid-habitable zone (as determined from its spectral type). With an assignment of $40 \%$ of the observing time available in a 5-year mission, SIM can probe the mid-habitable zone of 129 stars for $1.0 M_{\oplus}$ planets (Table 1). Over 450 stars can be observed in this same way to the $3.0 M_{\oplus}$ level. For an alternative strategy based on allocating equal time to all stars see Catanzarite et al. (2006).

The discovery space (planet mass vs. orbit radius) for the "Earth Analog Survey" is shown in Figure 1a, with the 129 stars filling a band in the lower portion of the plot. Distributing the observing time over a larger target list allows one to detect more terrestrial planets, albeit at higher masses. Table 1 shows the expected SIM yield for three different values of the search depth. A survey to a sensitivity of $3 M_{\oplus}$ would encompass more nearby stars than would likely be observed by the Terrestrial Planet Finder (TPF) Mission (Turnbull \& Tarter 2003). In each survey, the mass sensitivity improves with orbit radius, out as far as orbits with periods $\lesssim 5$ yr (Fig. 1a).

Based on assumptions discussed in Unwin et al. (2007) and including estimates of the incidence of planets of various masses and orbital radii from Cumming et al. (2007), Figure 2a shows histograms for the input vs detected numbers of terrestrial planet masses in the "Earth Analog" survey. The histogram shows counts based on statistics of 1000 simulated surveys. In the habitable zone, SIM would detect $61 \%$ of all the terrestrial planets, including almost half all planets with masses in the range $1-1.5 M_{\oplus}$, and nearly every planet of higher mass.

\section{The SIM Broad Survey}

In a "Broad Survey" using about 4\% of a 5-year mission, some 2000 stars will be measured 100 times each with a single measurement accuracy of $4 \mu$ as. This census will 

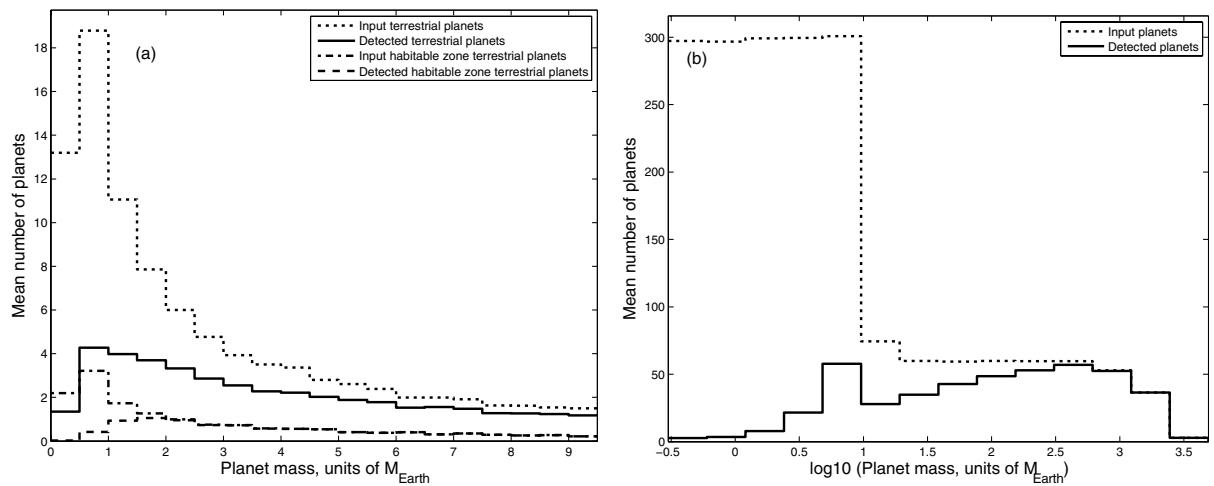

Figure 2. Histograms of the expected yield of terrestrial (a, left) and icy/gas giant (b, right) planets for the SIM Earth Analog and Broad Surveys. The histograms are the mean of 1000 simulated surveys in which geometric parameters of the model orbits were randomized. The mean input distributions of terrestrial $\left(M<10 M_{\oplus}\right)$ and giant planets are shown as dotted curves, and the mean number of planets detected by SIM as solid curves. For terrestrial planets in the habitable zone, the corresponding curves are shown as dash-dotted and dashed respectively.

include over 2000 stars of all spectral types (including O,B,A and early F, which are not accessible to RV measurements), binary stars, stars with a broad range of age and metallicity, stars with dust disks, evolved stars, white dwarfs, and stars with planets discovered with RV surveys. Each class addresses specific features of the planet-formation process: Are metals necessary for giant planet formation? Does the number of planets decline slowly with time due to dynamical evolution? What is the relation between dust disks and planets?. The Broad Survey is expected to yield a large sample of hot, cold, rocky, ice giant and gas giant planets, as well as multiple-planet systems for tests of planet-formation theories. Figure $2 \mathrm{~b}$ shows that relative to the assumed input population of planets (Unwin et al. 2007) SIM's Broad Survey will find $7 \%$ of the terrestrial planets, $2 \%$ of all terrestrial planets in the habitable zone, $47 \%$ of the Neptune-class planets and $87 \%$ of the Jupiter-class planets.

\section{The SIM Young Planets survey}

The "Young Planets" survey, targeted toward 150-200 stars with ages from 1 Myr to $100 \mathrm{Myr}$, will help us understand the formation and dynamical evolution of gas giant planets. The vast majority of the known planetary host stars are mature main sequence stars chosen for quiescent photospheres suitable for sensitive RV measurements. Similarly, stellar photospheres must be quiescent at the milli-magnitude level for transit detections. Since young stars have RV fluctuations or rotationally broadened line widths of hundreds of $\mathrm{m} \mathrm{s}^{-1}$ and brightness fluctuations of many percent, optical RV or transit observations cannot be used to detect planets around young stars. While near-IR RV measurements may detect a few "hot Jupiters" and coronagraphic imaging may find a few distant Jupiters $(>25 \mathrm{pc})$, it is a fact that as a result of the (irreducible) sensitivity limits of these other techniques, we know almost nothing about the incidence of planets within the region where these objects are actually thought to be forming. SIM will take advantage of the fact that a Jupiter orbiting at $1 \mathrm{AU}$ around a $0.8 M_{\odot}$ star at the distance of the youngest stellar associations (1-10 Myr) such as Taurus (140 pc) and Chamaeleon would produce an astrometric amplitude of $8 \mu$ as and up to $20 \mu$ as at the $25-50$ pc distance 


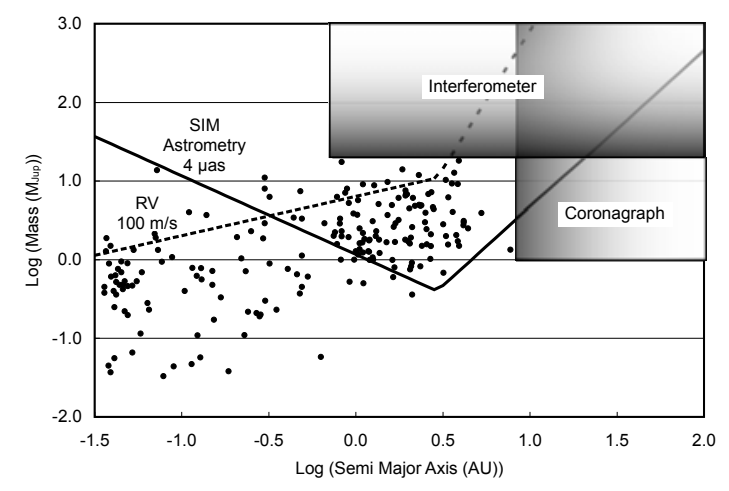

Figure 3. Planet mass detection sensitivity for the SIM Young Planet Survey (solid curve) in $\mathrm{M}_{J}$ versus orbital semi-major axis. Estimated capabilities for a large ground-based coronagraph (taken to be a diameter $d=30 \mathrm{~m}$ telescope at $\lambda=1.6 \mu \mathrm{m}$ operating an angular distance of $4 \lambda / d)$ and a near-IR interferometer $(85-\mathrm{m}$ baseline at $\lambda=1.6 \mu \mathrm{m})$ are shown as shaded boxes. Also plotted are the properties of the known radial velocity (RV) detected planets (dots). The $\mathrm{RV}$ accuracy for young stars (dashed curve) is limited to about $100 \mathrm{~m} \mathrm{~s}^{-1}$. The astrometric and imaging sensitivity limits assume a distance of $140 \mathrm{pc}$.

of the nearest young stars (10-50 Myr) such as members of the $\beta$ Pic and TW Hya groups. While some of the closest planetary systems will be detectable by Gaia, many of the youngest stars $(<10 \mathrm{Myr})$ will require SIM's measurement accuracy. Figure 3 shows SIM's expected astrometric accuracy for the SIM "Young Planet Survey" as a function of planet mass and semi-major axis.

A SIM survey of 200 young stars could find anywhere from 10-20 (assuming that only the presently known fraction of stars, 5-10\%, have planets) to 200 (all young stars have planets) planetary systems. These observations, when combined with the results of planetary searches of mature stars, will allow us to test theories of planetary formation and early Solar System evolution. By searching for planets around pre-main sequence stars carefully selected to span an age range from 1 to $100 \mathrm{Myr}$, we will learn at what epoch and with what frequency giant planets are found at the water-ice 'snowline' where they are expected to form (Pollack et al. 1996). This will provide insight into the physical mechanisms by which planets form and migrate from their place of birth, and about their survival rate.

Young stars are photospherically more active than main sequence stars, magnifying the concern expressed about starspots cited above for searches for terrestrial planets. A Monte Carlo analysis based on a starspot model (Beichman et al. 2001; Tanner et al. 2007) shows that for a typical T Tauri star radius of $3 R_{\odot}$, the astrometric jitter is less than $3 \mu$ as for an R-band variability less than 0.05 mag. A selection of target stars and a precursor observing program to validate them (Tanner et al. 2007) has identified more than 75 stars well suited for observation by SIM out of an initial target list of 200 objects; variability greater than 0.1 mag removed many of the potential targets. More stars will be added to the precursor program to bring the total up to the desired number of 150-200 stars. SIM will make 100 visits to each star (up to 50 2-D visits) which, spread over 5 years, will be adequate to identify and characterize up to 3 planets per star having periods ranging from less than a year up to 2.5 years. 


\section{Conclusions}

We have described the compelling use of the Space Interferometer Mission (SIM Planetquest) for the detection of planets beyond our solar system. SIM can survey over 100 nearby stars for $1 \mathrm{M}_{\oplus}$ planets in the habitable zone of their parent stars and over 300 stars at a sensitivity adequate to find $3 \mathrm{M}_{\oplus}$ planets. A descoped version of SIM, SIMLite, will be able to achieve these same goals around a smaller number of stars. No other mission is technologically ready to pursue such dramatic goals along the path to identifying other habitable planets around nearby solar analogs. SIM can lead the way for direct detection missions such as the Terrestrial Planet Finder and Darwin, identifying specific host stars and target planets, as well as providing essential information such as mass needed for detailed characterization. In addition to its unique role in finding and characterizing terrestrial planets, SIM will make unique contributions to the study of gas and icy planets around a wide variety of stars as well as enabling a study of newly formed planets that may help to unravel the processes of planetary system formation.

\section{Acknowledgments}

We gratefully acknowledge the contributions of the many dedicated scientists, technologists, engineers and managers. who have worked on SIM over the years. The research described in this paper was carried out at the Jet Propulsion Laboratory, California Institute of Technology, under contract with the National Aeronautics and Space Administration. This research has made use of the NASA/IPAC Infrared Science Archive, which is operated by the Jet Propulsion Laboratory, California Institute of Technology, under contract with the National Aeronautics and Space Administration.

\section{References}

Beaulieu, J.-P. et al. 2006, Nature, 439, 437.

Beichman, C. A., Fridlund, M., Traub, W. A., Stapelfeldt, K. R., Quirrenbach, A., Seager, S. 2007 in Protostars and Planets V, B. Reipurth, D. Jewitt, and K. Keil (eds.), University of Arizona Press, Tucson, p. 915.

Beichman, C. A. 2001, in Young Stars Near Earth: Progress and Prospects, ASP Conference Series Vol. 244. Edited by Ray Jayawardhana and Thomas Greene. San Francisco: Astronomical Society of the Pacific, p. 376.

Butler, R. P., Wright, J. T., Marcy, G. W., Fischer, D. A., Vogt, S. S., Tinney, C. G., Jones, H. R. A., Carter, B. D., Johnson, J. A., McCarthy, C., \& Penny, A. J. 2006, ApJ, 646, 505

Catanzarite, J., Shao, M., Tanner, A., Unwin, S., \& Yu, J. 2006, PASP, 118, 1322

Cumming, A., Marcy, G. W., Butler, R. P., et al. 2007, ApJ, in preparation

Frink, S., Quirrenbach, A., Fischer, D., Röser, S., \& Schilbach, E. 2001, PASP, 113, 173

Henry, G. W., Marcy, G. W., Butler, R. P., Vogt, S. S. 2000, ApJ, 529, L41.

Ida, S., \& Lin, D. N. C. 2005, ApJ, 626, 1045

Kasting, J. F., Whitmire, D. P., Reynolds, R. T. 1993, Icarus, 101, 108.

Marcy, G., Butler, R. P., Fischer, D., Vogt, S., Wright, J. T., Tinney, C. G., \& Jones, H. R. A. 2005, Progress of Theoretical Physics Supplement, 158, 24

Mayor, M. \& Queloz, D. 1995, Nature, 378, 355.

Pollack, J., Hubickyj, O., Bodenheimer, P., Lissauer, J. J., Podolak, M. Greenzweig, Y. 1996, Icarus, 124,62

Struve, O. 1952, The Observatory, 72, 199.

Tanner, A., et al. 2007, PASP, 119, 747

Turnbull, M. C., \& Tarter, J. C. 2003, $A p J(S), 145,181$

Unwin, S. C., and the SIM Science Team 2007, PASP, in press. 